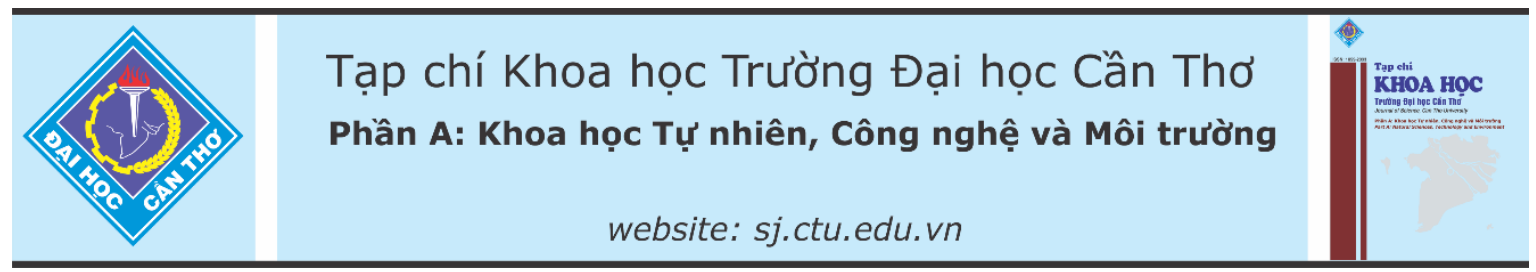

DOI:10.22144/ctu.jvn.2021.034

\title{
ÚNG DỤNG CÔNG NGHỆ UAV (DRONES) THEO DÕI VÀ HỖ TRỢ CẢNH BÁO SỚM DỊCH HẠI VÙNG CANH TÁC LÚA TỈNH SÓC TRĂNG
}

\author{
Võ Quốc Tuấn ${ }^{1 *}$, Nguyễn Tấn Lợi ${ }^{1}$, Quãng Thị Dal ${ }^{2}$, Trương Chí Quang ${ }^{1}$ và Phạm Quốc Việt ${ }^{3}$ \\ ${ }^{1}$ Bộ môn Tài nguyên đất đai, Khoa Môi trường và Tài nguyên Thiên nhiên, Truờng Đại học Cần Thơ \\ ${ }^{2}$ Sở Tài nguyên và Môi truoòng tỉnh Kiên Giang \\ ${ }^{3}$ Hoc viên cao họ ngành quản lý đai khóa 27 , Truòng Đại hoc Cần Tho \\ *Nguoòi chịu trách nhiệm về bài viết:Võ Quốc Tuấn (email: vqtuan@ctu.edu.vn)
}

\section{Thông tin chung:}

Ngày nhận bài: 15/09/2020

Ngày nhận bài sưa: 13/11/2020

Ngày duyệt đăng: 28/04/2021

Title:

Application of UAV (drones) technology for monitoring and early warning of pests in rice cultivated area in Soc Trang province

\section{Tù̀ khóa:}

Cảnh báo sớm, dịch hại, UAV, viễn thám

\section{Keywords:}

Early warning, pests and diseases, remote sensing, $U A V$

\begin{abstract}
The Mekong Delta, often referred to as "Vietnam's Rice Bowl", is the biggest rice producing region in Vietnam with the majority of its land area used for cultivating paddy rice. However, the intensification of rice cultivation over many years has produced more pests and diseases. The research is conducted to monitor and support early warning of rice pests and diseases using unmanned aerial vehicle (UAV) technology. The researcher did analyze the relationship between the level of pest infestation and the values of Normalized Difference Vegetation Index (NDVI), Normalized Difference Red Edge Index (NDRE - Normalized Difference Red Edge Index), and field survey data. The results show that there are 4 levels of pest infestations: heavy, medium, light and no pest infection with a total infected area of $11.37 \mathrm{ha}$, in which heavy infection is 2.1 ha, medium infection is 2.76 ha, light infection is 6.51ha and no infection accounts for $12.33 \mathrm{ha}$. It shows that there is potential to apply UAV technology in monitoring rice pests and diseases, which contributes to improve the efficiency of rice production in Soc Trang province in particular and in the Mekong River Delta in general.
\end{abstract}

\section{TÓM TẮT}

Đồng bằng sông Cửu Long là vùng canh tác lúa trọng điểm của cả nuớc, tuy nhiên việc thâm canh tăng vu trong nhiều năm đã làm cho tình hình sâu bệnh diễn biến phức tạp. Nghiên cứu được thực hiện nhằm ứng dụng công nghệ máy bay không người lái (UAV - unmanned aerial vehicle) để theo dõi và cảnh báo sớm dịch hại. Nghiên cứu phân tích mối quan hệ giữa mức độ nhiếm dịch hại trên lúa dựa trên chi số khác biệt thực vật (NDVI - normalized difference vegetation index), chi số khác biệt rìa đỏ (NDRE - normalized difference red edge index), và số liệu điều tra thưc địa được thu thâp tại thời điểm chup ảnh. Kết quả phân tích đã phân loại được 4 mức độ nhiếm dịch hại trên lúa: nhiếm dịch hại nặng, nhiếm dịch hại trung bình, nhiễm dịch hại nhe và không nhiếm dịch hai với tổng diện tích nhiếm là 11,37 ha. Trong đó, nhiếm nặng chiếm 2,1 ha, nhiễm trung bình chiếm 2,76 ha, nhiếm nhe chiếm 6,51 ha và không nhiếm là 12,33 ha. Qua đó cho thấy khả năng ứng dụng công nghệ UAV trong theo dõi và hỗ trợ cảnh báo sớm dịch hại trên cây lúa mang lại nhiều hiệu quả, góp phần nâng cao hiệu quả sản xuất lúa tại tỉnh Sóc Trăng nói riêng và vùng Đồng bằng sông Cưu Long nói chung. 


\section{GIỚI THIÊU}

Ngày nay, việc sử dụng thiết bị bay không người lái đang được sử dụng rộng rãi trong lĩnh vực nông nghiệp (Sinha et al., 2016; Kameyama \& Sugiura, 2020), một số nghiên cứu ứng dụng gần đây như ứng dụng công nghệ UAV để phát hiện sớm dịch hại trên vườn nho, bọ cánh cứng và cỏ dại trên cà rốt và hành tây (Bouroubi et al., 2017); ứng dụng UAV để phát hiện sớm dịch hại, theo dõi sức khỏe và hàm lượng chất dinh dưỡng trên cây trồng (Filho et al., 2019; Matese et al., 2015). Đặc biệt trên cây lúa, UAV được sử dụng trong giám sát giai đoạn đầu tăng trưởng của lúa bằng máy ảnh kỹ thuật số đa năng Red, Green, Blue (RGB) (Norasma et al., 2018); sử dụng UAV trong ước tính năng suất lúa (Duan et al., 2019), ứng dụng UAV trong theo dõi quá trình tăng trưởng lúa thực nghiệm giống lúa (Chosa et al., 2010); thành lập hệ thống tạo bản đồ và phát hiện sớm dịch hại trên lúa bằng Drone và IoT (Kitpo \& Inoue, 2018).

Dữ liệu ảnh UAV cho phép phát triển nhanh chóng và liên tục các công cụ giám sát, quản lý cây trồng, giúp phát hiện sớm dịch hại và côn trùng. Vì vậy, UAV là thiết bị tiềm năng cho việc thực hiện nhiệm vụ này, dòng sản phẩm DJI Inspire 2, mang cảm biến nông ngiệp Sentera Double 4K (Chỉ số khác biệt thực vật chuẩn hóa - NDVI và chỉ số khác biệt rìa đỏ - NDRE) là công cụ phù hợp cho theo dõi sự phát triển của lúa, với kích thước điểm ảnh nhỏ nên dễ dàng nhận dạng các đối tượng dịch hại (Norasma et al., 2019). Hơn nữa, việc chủ động thời gian bay phù hợp với các giai đoạn phát triển của cây lúa, giúp thực hiện các biện pháp phòng ngừa, ngăn chặn sự lây lan dịch hại kịp thời (Aylor et al., 2006; Hunt et al., 2003; Strange \& Scott, 2005; Swain et al., 2007).
Do đó, việc ứng dụng công nghệ UAV được xem là công cụ tìm năng cho việc theo dõi và hỗ trợ cảnh báo sớm dịch hại trên lúa, với khả năng cung cấp dữ liệu kịp thời, với độ phân giải không gian rất cao, kết hợp với cảm biến nông nghiệp, giúp phát hiện sớm dịch hại, đảm bảo thời gian thực hiện các biện pháp phòng ngừa, ngăn chặn sự lây lan và giảm thiểu các tác động xấu cho sản xuất lúa. Vì thế, nghiên cứu được thực hiện nhằm đánh giá khả năng ứng dụng công nghệ UAV trong theo dõi và hỗ trợ cảnh báo sớm dịch hại trên vùng canh tác lúa tại tỉnh Sóc Trăng.

\section{PHƯƠNG PHÁP THỬC HIÊN}

\subsection{Thu thập dữ liệu và điều tra khảo sát thực địa}

\subsubsection{Thu thập dĩ liệu ảnh $U A V$}

Dữ liệu ảnh UAV được thu thập bởi thiết bị DJI Inspire 2, sử dụng cảm biến Sentera Double $4 \mathrm{~K}$ được thiết kế đặc biệt dùng trong lĩnh vực nông nghiệp, có khả năng ghi lại 3 kênh phổ (kênh phổ giả DNred, DNGreen, DNBlue) dưới định dạng *.jpg, trong đó có 2 kênh phổ được sử dụng để tính toán các chỉ số thực vật (NDVI và NDRE). Ảnh này cần phải thực hiện một số phép tính (Band math) để tách các kênh phổ DNRed, DNGreen, DNBlue gốc thành kênh phổ Đỏ (Red), kênh phổ rìa Đỏ (RedEdge) và kênh phổ cận hồng ngoại (NIR).

Nguồn dữ liệu ảnh thu được có độ phân giải không gian là $4,62 \mathrm{~cm}$, với độ phủ $70 \%$ (side overlap), che phủ 23,7 ha diện tích lúa tại xã Tân Hưng, huyện Long Phú, tỉnh Sóc Trăng, được thể hiện trong Hình 1. Dữ liệu ảnh UAV được chụp ngày $22 / 11 / 2018 ; 05 / 12 / 2018 ; 12 / 12 / 2018$ và 25/01/2019 với 694 ảnh, được trình bày trong Bảng 1.

Bảng 1. Thời gian và số lượng ảnh thu thập

\begin{tabular}{lcccccccc}
\hline & \multicolumn{2}{c}{$\mathbf{2 2 / 1 1 / 2 0 1 8}$} & \multicolumn{2}{c}{$\mathbf{0 5 / 1 2 / 2 0 1 8}$} & \multicolumn{2}{c}{$\mathbf{1 2 / 1 2 / 2 0 1 8}$} & \multicolumn{2}{c}{$\mathbf{2 5 / 0 1 / 2 0 1 9}$} \\
\cline { 2 - 8 } & NDVI & NDRE & NDVI & NDRE & NDVI & NDRE & NDVI & NDRE \\
\hline Tuối lúa & \multicolumn{2}{c}{30 ngày } & \multicolumn{2}{c}{43 ngày } & \multicolumn{2}{c}{50 ngày } & \multicolumn{1}{c}{ Thu hoạch } \\
Số lượng ảnh & 183 & 183 & 176 & 176 & 143 & 143 & 194 & 194 \\
\hline
\end{tabular}




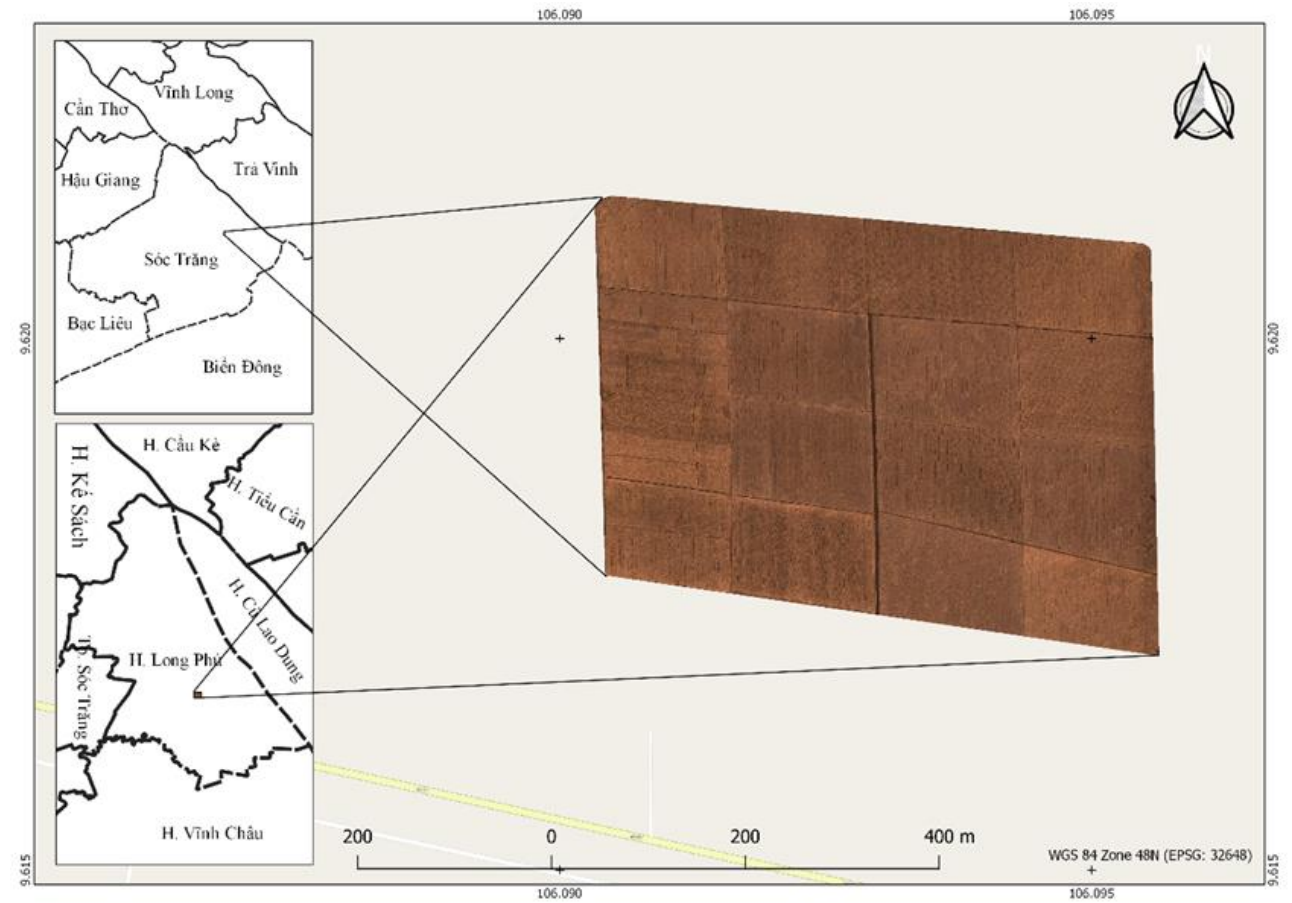

Hình 1. Bản đồ vùng nghiên cứu

\subsection{2. Điều tra, khảo sát thục địa}

Nghiên cứu thực hiện khảo sát tại 15 điểm trong khu vực nghiên cứu, số liệu về hiện trạng sâu bệnh được mô tả bởi chuyên gia tại Chi cục Trồng trọt và Bảo vệ thực vật ở địa phương, được thực hiện theo quy chuẩn Việt Nam năm 2014 về "Về phương pháp điều tra phát hiện dịch hại lúa" của Bộ Nông nghiệp và Pháp triển Nông thôn (QCVN 01-166: 2014/BNNPTNT).

Thời gian khảo sát thực địa được thực hiện cùng lúc với thời gian thu thập ảnh. Đối tượng điều tra là các yếu tố đại diện có liên quan đến dịch hại, bao gồm thông tin dịch hại: sâu bệnh, dịch bệnh; các thông tin về cây lúa: giống, thời vụ, giai đoạn sinh trưởng, phát triển của cây lúa và tập quán canh tác; kết hợp với thông tin địa lý như tọa độ và ảnh chụp hiện trạng mặt đất. Từ đó, tính toán, thống kê các thông số về mật độ, số lượng sâu bệnh, tỷ lệ dịch hại. Thông qua đó, phân cấp các mức độ nhiễm dịch hại: nhiễm nhẹ, nhiễm trung bình, nhiễm nặng và thống kê diện tích nhiễm được thực hiện theo QCVN 01-166: 2014/BNNPTNT.

\subsection{Xây dựng ảnh ghép trực giao (Orthomosaic)}

Quá trình xây dựng ghép ảnh trực giao (Orthomosaic) được thực hiện trên phần mềm Agisoft PhotoScan/Metashape. Mục đích của quá trình này dùng để ghép ảnh, sửa lỗi hình học, sự biến dạng hình học của ảnh ban đầu, quá trình xử lý càng chi tiết thì độ sai lệch hình học càng thấp, đảm bảo tính chính xác của ảnh cũng như vị trí của ảnh (Ngadiman et al., 2018).

Quy trình xử lý ảnh ghép trực giao bao gồm 5 bước chính: 1. Thêm ảnh (add photos), 2. Liên kết ảnh (align photos), 3. Xây dựng đám mây điểm dày đặc (build dense cloud), 4. Xây dựng mô hình số bề mặt (digital surface model - DSM), 5. Tạo ảnh ghép trực giao, quy trình thực hiện được thể hiện trong Hình 2. 


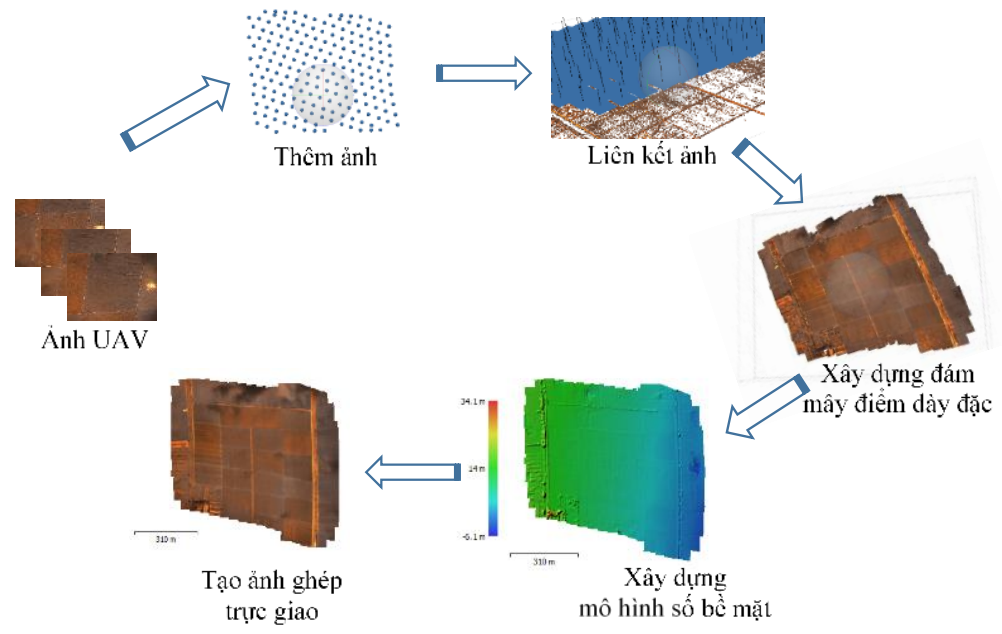

Hình 2. Quá trình xây dựng ảnh ghép trực giao

\subsection{Tạo chỉ số khác biệt thực vật (NDVI) và} chỉ số khác biệt rìa đỏ (NDRE)

Chỉ số khác biệt thực vật và chỉ số khác biệt rìa đỏ được thành lập bằng công cụ "Raster Calculator" trên phần mềm Qgis. Công thức tính chỉ số NDVI trên cảm biến Sentera Double $4 \mathrm{~K}$ sử dụng kênh xanh dương (Blue) cho kênh NIR và kênh đỏ (Red) cho kênh Red. Kênh xanh lá được loại bỏ khi thực hiện tính toán chỉ số và dải phổ.

Chỉ số NDVI được tính toán theo công thức sau:

$$
\text { Red }=-0,966 * \text { DNblue }+1,000 * \text { DNred }
$$

(MicaSense, 2018)

$$
\text { NIR }=4,350 * \text { DNblue }-0,286 * \text { DNred }
$$

(MicaSense, 2018)

$$
\text { NDVI }=\frac{(\text { NIR-Red })}{(\text { NIR }+ \text { Red })}
$$

(Tucker, 1979)

Công thức tính chỉ số NDRE gần giống như chỉ số khác biệt thực vật, nhưng sử dụng tỷ số giữa kênh phổ gần hồng ngoại và kênh phổ rìa đỏ (Barnes et al., 2000), được xác định bởi công thức sau:

RedEdge $=-0,956 *$ DNblue $+1,000 *$ DNred (MicaSense, 2018)

NIR $=2,426 *$ DNblue $-0,341 *$ DNred

(MicaSense, 2018)

$$
\mathrm{NDRE}=\frac{(\text { NIR }- \text { RedEdge })}{(\text { NIR }+ \text { RedEdge })} \quad \text { (Barnes et al., 2000) }
$$

\section{4. Đánh giá quan hệ giữa giá trị NDVI/NDRE với mức độ nhiễm dịch hại của lúa}

Sau khi chỉ số NDVI và NDRE được tạo thành, sử dụng công cụ "Point Sampling tool" trên phần mềm Qgis để trích xuất giá trị NDVI và NDRE tại 15 điểm khảo sát nhằm kết nối thông tin trên ảnh và hiện trạng nhiễm bệnh trên mặt đất. Đồng thời kết hợp phương pháp kiểm định Anova (kiểm định giữa biến định lượng "biến giá trị NDVI/NDRE" và biến định tính "biến mức độ nhiễm dịch hại trên lúa") trên phần mềm thống kê "IBM SPSS Statistics" để phân tích mối quan hệ giữa chỉ số NDVI và NDRE với mức độ lúa nhiễm dịch hại (Sow, 2014).

Dựa vào mức ý nghĩa (Significance) để xác định giữa giá trị NDVI/NDRE và mức độ lúa nhiễm dịch hại có mối quan hệ hay không, nếu hệ số Significance (Sig.) $\mathrm{p}<0,05$ thì xác định giữa giá trị NDVI/NDRE và mức độ nhiễm dịch hại có mối quan hệ; ngược lại $\mathrm{p}>0,05$ sẽ không xảy ra mối quan hệ. Thông qua hệ số ảnh hưởng (Eta Squared) giúp xác định mức độ mối hệ giữa giá trị NDVI/NDRE và mức độ nhiễm dịch hại (Sow, 2014).

\section{KẾT QUẢ THẢO LUÂN}

\subsection{Kết quả điều tra, khảo sát thực địa}

Kết quả khảo sát thực địa (dựa theo phương pháp điều tra QCVN 01-166: 2014/BNNPTNT) tại 15 điểm khảo sát, được thể hiện trong Hình 3 , Hình 4 cho thấy diện tích lúa bị ảnh hưởng bởi dịch hại nhiều nhất (sâu cuốn lá, bệnh đạo ôn và bệnh cháy lá) ở giai đoạn đẻ nhánh (30 ngày tuổi), trong đó tổng diện tích nhiễm bệnh 10,7 ha, chiếm $45,15 \%$, phần lớn các trà lúa nhiễm bệnh ở cấp độ nhẹ (6ha).

Ở các giai đoạn tiếp theo (lúa 43 ngày và 50 ngày tuổi) tình hình nhiễm bệnh có xu hướng giảm do dịch bệnh được kiểm soát và bổ sung diện tích lúa bị nhiềm nặng. Ở giai đoạn đẻ nhánh, sinh vật gây 
hại chính là sâu cuốn lá và bệnh đạo ôn lá, sâu cuốn lá phổ biến từ tuổi 1 đến tuổi 4 và bệnh đạo ôn đang gây hại tập trung nhiều ở các trà lúa cấy dặm với mức độ nhiễm từ trung bình đến nặng. Riểng đối tượng rầy nâu có mật độ dưới ngưỡng gây hại, chủ yếu là rầy nâu tuổi $2,3,4$ và rầy trưởng thành mang trứng.

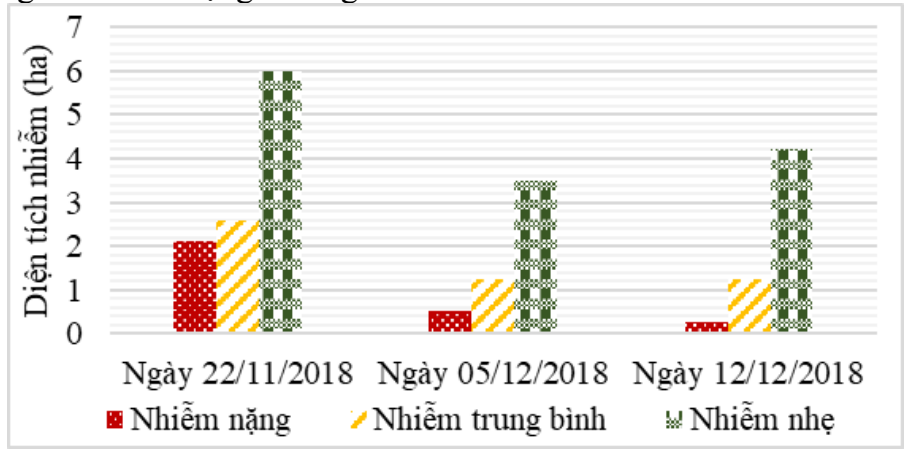

Hình 3. Biểu đồ diện tích lúa nhiễm dịch hại

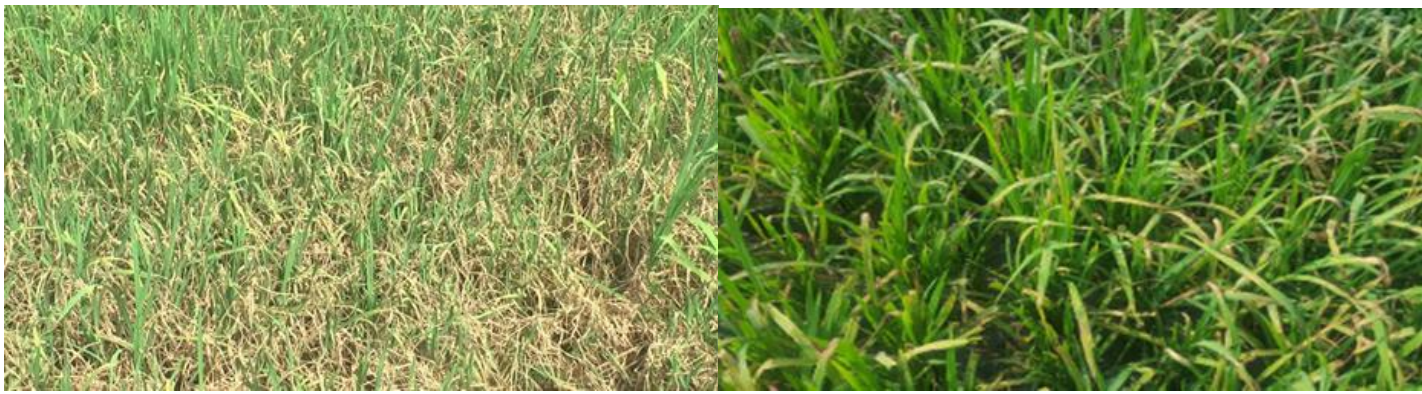

a) Lúa bị nhiễm nặng

b) Lúa bị nhiễm trung bình

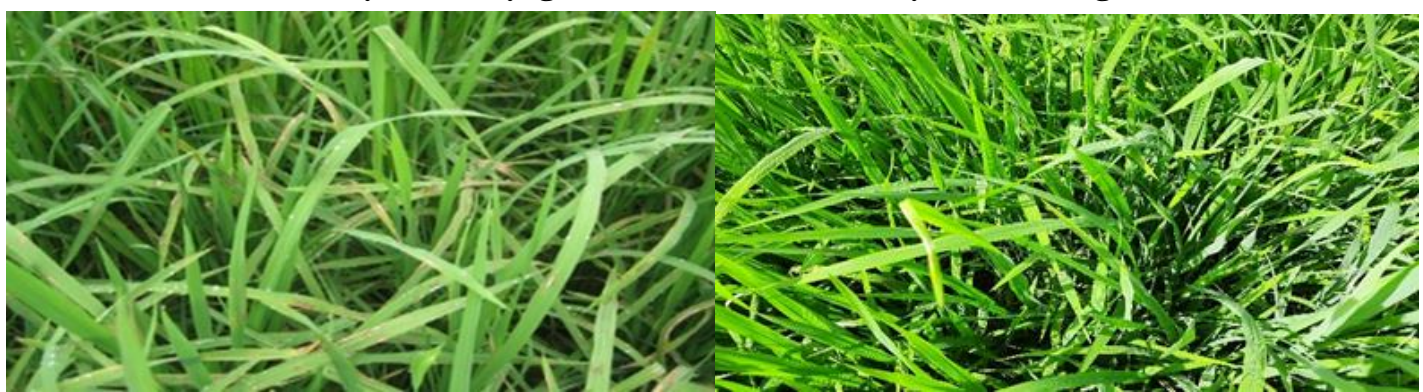

c) Lúa bị nhiễm nhẹ

d) Lúa không bị nhiễm

\section{Hình 4. Mức độ nhiễm dịch hại trên lúa}

\subsection{Kết quả xây dựng ảnh ghép trực giao}

Ảnh ghép trực giao được xây dựng thông qua việc kết nối các ảnh riêng lẻ được thu thập bởi thiết bị UAV tạo thành ảnh trên toàn bộ nghiên cứu, giúp cho quá trình tính toán và phân loại sau này được thuận tiện và dễ dàng hơn. Hơn nữa, do dữ liệu ảnh có độ trùng lặp cao, nên các điểm ảnh bị lỗi được thay thế bằng các điểm ảnh có chất lượng tốt hơn, vì thế ảnh sau khi xử lý có độ chính xác cao hơn.
Màu của ảnh ghép trực giao được thể hiện thông qua ảnh màu giả cận hồng ngoại (NIR) được tổ hợp từ 3 kênh phổ DNRed, DNGreen, DNBlue, được thể hiện trong Hình 5 . Trong đó, các đối tượng có nhiều thực vật thì có màu càng đậm, ngược lại càng ít thực vật thì có màu càng hạt. Tuy nhiên, để có thể nhận dạng rõ các đối tượng thì cần thực hiện phân loại hiện trạng mặt đất thông qua các chỉ số thực vật như NDVI và NDRE. 


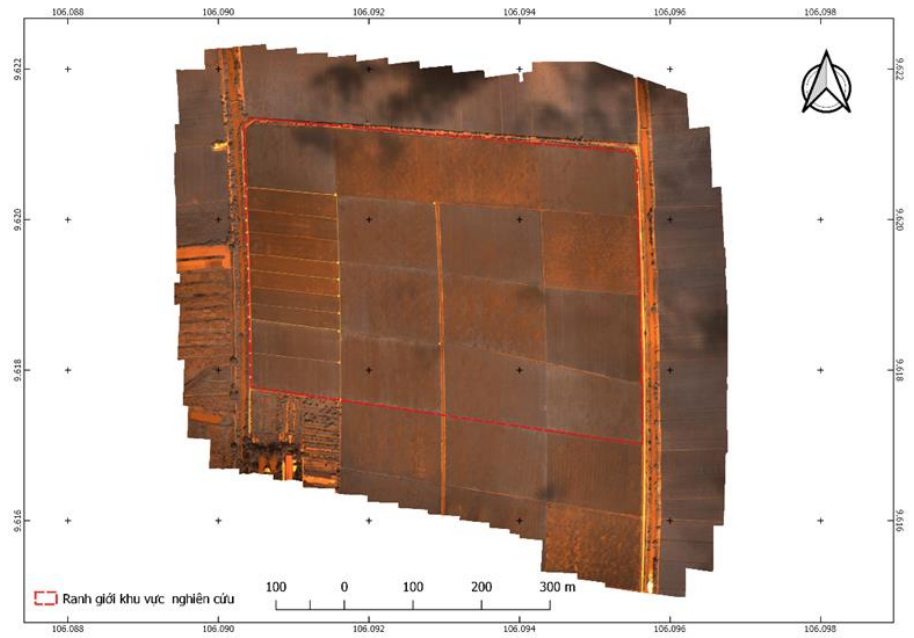

Hình 5. Kết quả xây dụng ảnh ghép trực giao (chụp ngày 22/11/2018)

3.3. Kết quả đánh giá quan hệ giữa chỉ số NDVI và NDRE với mức độ nhiễm dịch hại của lúa

3.3.1. Kết quả theo dõi giá trị NDVI và NDRE tại các điểm lúa bị nhiếm dịch hại

Kết quả theo dõi chuỗi giá trị NDVI trong giai đoạn nghiên cứu được trình bày trong Hình 6 , Hình 7 , Hình 8 và Hình 9 cho thấy lúa không nhiễm dịch hại trong giai đoạn 30 ngày tuổi có giá trị NDVI lớn hơn 0,5 và ngược lại khi nhiễm dịch hại thì lúa có giá trị NDVI sẽ thấp hơn 0,5 (ở mức độ nhiễm nhẹ) và thấp hơn 0,4 (ở mức độ nhiễm nặng và trung bình). Tương tự ở giai đoạn 43 ngày và 50 ngày, khi lúa không bị nhiễm dịch hại có giá trị NDVI lần lượt lớn hơn 0,6 và 0,62 và ngược lại khi lúa bị nhiễm dịch hại sẽ có giá trị NDVI từ $-0,5$ đến thấp hơn 0,6 và 0,62 . Khi lúa bị nhiễm bệnh thì giá trị NDVI sẽ đột nhiên giảm thấp do sự phát triển mạnh của dịch hại làm cho hàm lượng diệp lục tố trên thân và lá giảm, vì thế giá trị NDVI có xu hướng giảm theo. Tuy nhiên, do có sự ngăn chặn và kiểm soát dịch hại kịp thời nên các trà lúa nhiễm dịch hại ở mức độ trung bình và nhẹ dần dần phục hồi và tăng trưởng trở lại ở các giai đoạn sau.

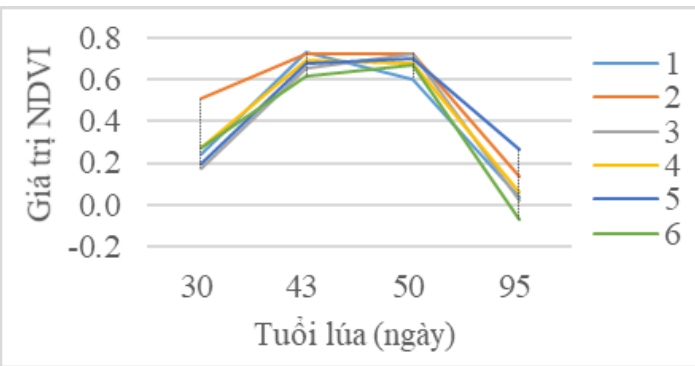

Hình 6. Biểu đồ thay đổi giá trị NDVI tại các điểm (1-6) nhiễm bệnh giai đoạn lúa 30 ngày tuổi

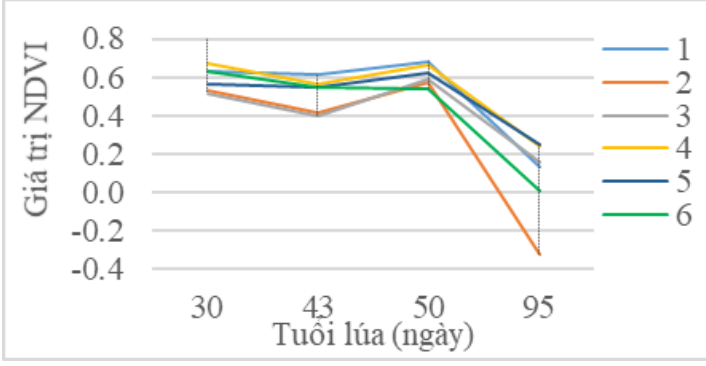

Hình 7. Biểu đồ thay đổi giá trị NDVI tại các điểm (1-6) nhiễm bệnh giai đoạn lúa 43 ngày tuổi

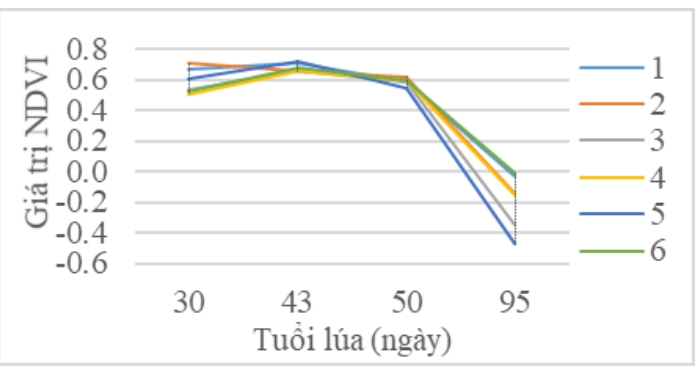

Hình 8. Biểu đồ thay đổi giá trị NDVI tại các điểm (1-6) nhiễm bệnh giai đoạn lúa 50 ngày tuổi

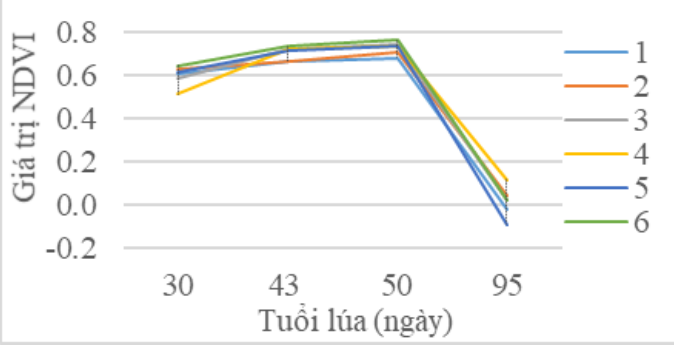

Hình 9. Biểu đồ thay đổi giá trị NDVI tại các điểm (1-6) lúa không bị nhiễm bệnh 
Kết quả nghiên cứu chuỗi giá trị NDRE được thể hiện trong Hình 10, Hình 11, Hình 12 và Hình 13 cho thấy biểu đồ thay đổi giá trị NDRE cũng tương tự như giá trị NDVI, lúa không bị nhiễm dịch hại có giá trị NDRE tăng dần dần theo giai đoạn tăng trưởng và ngược lại lúa khi bị nhiễm dịch hại thì giá trị NDRE sẽ đột ngột bị giảm xuống. Tuy nhiên, các ngưỡng giá trị NDRE ở các mức độ nhiễm bệnh thấp hơn ngưỡng giá trị NDVI, cụ thể: trong giai đoạn lúa 30 ngày tuổi, lúa không bị nhiễm dịch hại có giá trị NDRE lớn hơn 0,05 và lúa nhiễm dịch hại có giá trị NDRE trong khoảng từ $-0,5$ đến 0,05 ; tương tự ở giai đoạn lúa 43 ngày và 50 ngày tuối thì ngưỡng giá trị NDRE của lúa nhiễm dịch hại tương ứng trong khoảng là từ $-0,5$ đến 0,07 và từ $-0,5$ đến 0,1 .

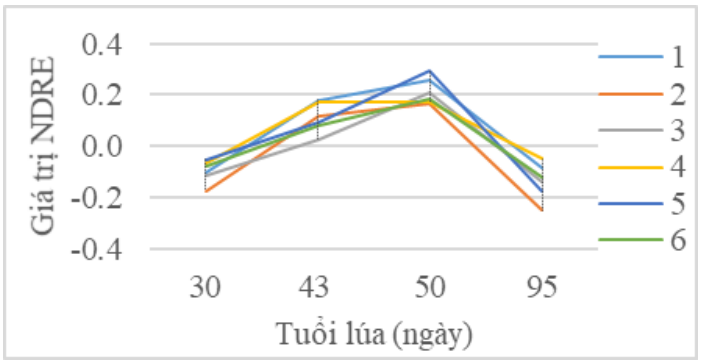

Hình 10. Biểu đồ thay đổi giá trị NDRE tại các điểm (1-6) nhiễm bệnh giai đoạn lúa 30 ngày

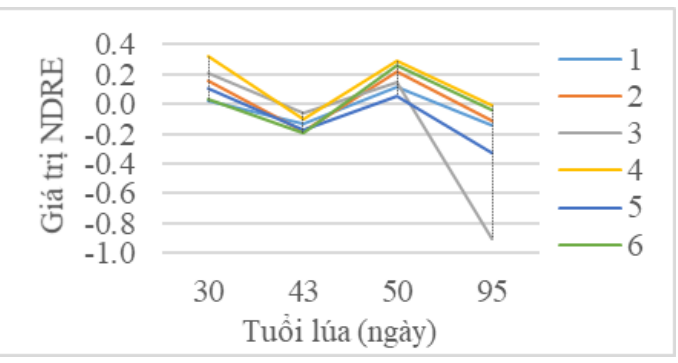

Hình 11. Biểu đồ thay đổi giá trị NDRE tại các điểm (1-6) nhiễm bệnh giai đoạn lúa 43 ngày

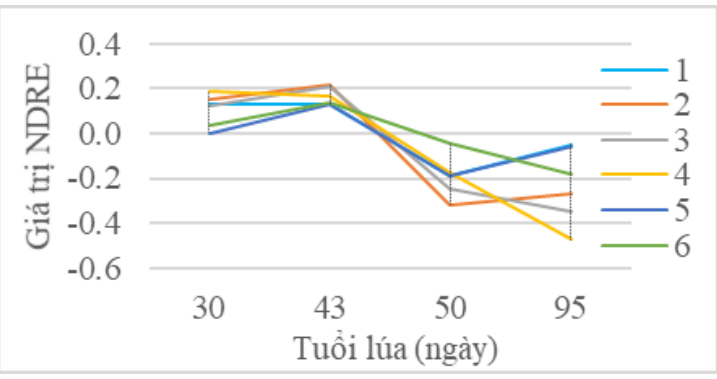

Hình 12. Biểu đồ thay đổi giá trị NDRE tại các điểm (1-6) nhiễm bệnh giai đoạn lúa 50 ngày

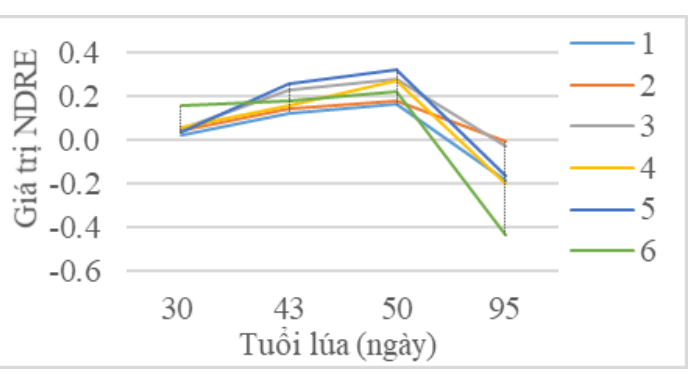

Hình 13. Biểu đồ thay đổi giá trị NDRE tại các điểm (1-6) lúa không bị nhiễm bệnh

3.3.2. Kết quả đánh giá quan hệ giữa chi số NDVI và NDRE với mức độ nhiếm dịch hại của lúa

Kết quả đánh giá quan hệ giữa chỉ số NDVI và NDRE với mức độ nhiễm dịch hại, được thể hiện trong Bảng 2 cho thấy, chỉ số NDVI và mức độ lúa nhiễm dịch hại có mối quan hệ với nhau, với mức ý nghĩa (significance) Sig. $\approx 0,0001<0,05$. Hệ số ảnh hưởng (Eta Squared) giữa mức độ lúa nhiễm bệnh và giá trị NDVI tương đối cao $\left(\eta^{2}=0,717\right)$, qua đó cho thấy có $71,7 \%$ hiện trạng lúa nhiễm bệnh tương ứng với giá trị NDVI trên ảnh. Thông qua Bảng 2; biểu đồ Hình 6 , Hình 7, Hình 8 và Hình 9 nhận thấy, giá trị NDVI càng thấp tương ứng với mức độ lúa nhiễm bệnh càng nặng.

Kết quả đánh giá mối quan hệ giữa chỉ số NDRE và mức độ lúa nhiễm dịch hại, được trình bày trong Bảng 2 cũng cho thấy chỉ số NDRE và mức độ lúa nhiễm dịch hại có mối quan hệ với nhau với hệ số Sig. $\approx 0,0001<0,05$. Hệ số ảnh hưởng giữa giá trị NDRE và mức độ lúa nhiễm dịch hại cao hơn hệ số ảnh hưởng giữa giá trị NDVI và mức độ nhiễm dịch hại, với hệ số ảnh hưởng $\eta^{2}=0,831$. Thông qua kết quả đánh giá mối quan hệ giữa giá trị NDVI và NDRE với mức độ nhiễm dịch hại của lúa, thấy rằng chỉ số NDRE phản ánh chính xác mức độ nhiễm dịch hại của lúa hơn chỉ số NDVI.

Bảng 2. Kết quả đánh giá quan hệ giữa giá trị NDVI/NDRE với hiện trạng khảo sát lúa nhiễm dịch hại

\begin{tabular}{lcc}
\hline $\begin{array}{l}\text { Giá trị mối } \\
\text { quan hệ }\end{array}$ & $\begin{array}{c}\text { Mức ý nghĩa } \\
\text { (Significance) }\end{array}$ & $\begin{array}{c}\text { Hệ số ảnh hưởng } \\
\text { (Eta Squared) }\end{array}$ \\
\hline Giá trị NDVI & 0,0001 & 0,717 \\
Giá trị NDRE & 0,0001 & 0,831 \\
\hline
\end{tabular}

\subsection{Kết quả phân tích diễn biến dịch hại trên lúa}

Kết quả phân tích diễn biến dịch hại thông qua chỉ số NDVI được trình bày trong bản đồ Hình 14 và biểu đồ Hình 16 cho thấy lúa bị nhiễm dịch hại nghiêm trọng nhất ở giai đoạn 30 ngày tuổi với hơn 
12,33 ha nhiễm, chiếm hơn 52,03\%. Trong đó, diện tích nhiễm từ trung bình đến nặng chiếm 3,02 ha, chủ yếu do ảnh hưởng bởi sâu lá và bệnh đạo ôn.

Trong các giai đoạn lúa 43 ngày và 50 ngày tuổi diện tích nhiễm dịch hại suy giảm đáng kể, trong đó

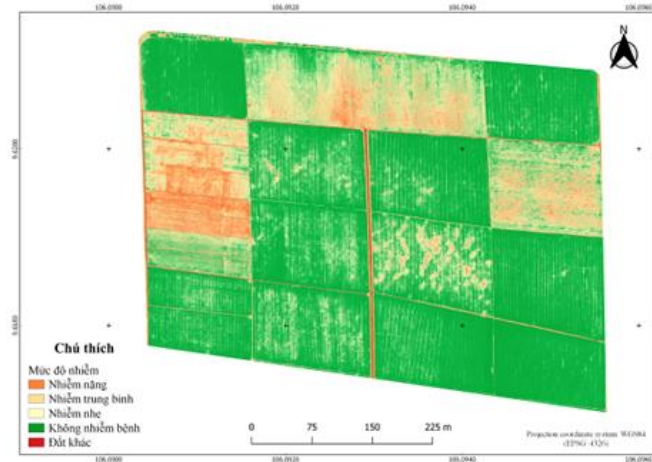

a) Bản đồ lúa bị nhiễm bệnh ngày 22/11/2018

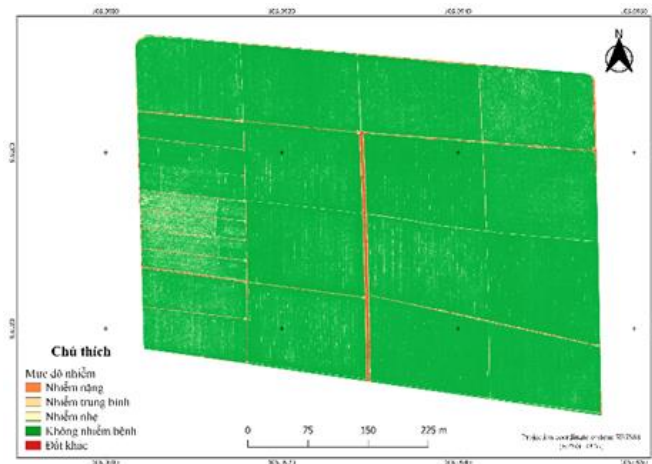

c) Bản đồ lúa bị nhiễm bệnh ngày 12/12/2018 tổng diện tích nhiễm chỉ còn tương ứng là 0,48 ha và 0,61 ha chiếm $2 \%$ và $2,6 \%$. Phần lớn diện tích lúa nhiễm bệnh ở mức độ trung bình và nhẹ, lúa chủ yếu bị ảnh hưởng bởi sâu lá, phân bố đều trên toàn bộ khu vực nghiên cứu.

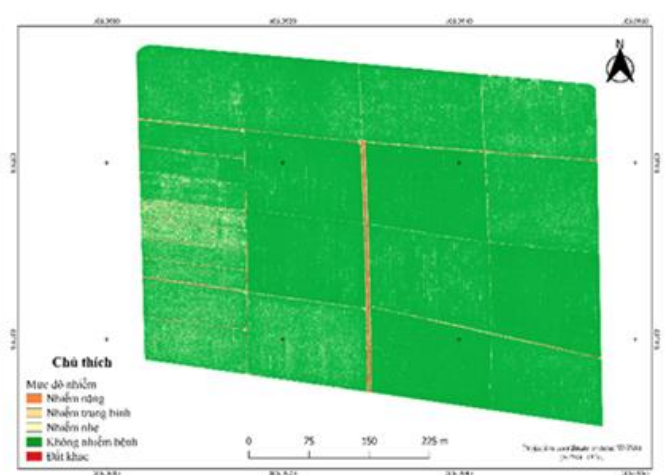

b) Bản đồ lúa bị nhiễm bệnh ngày 5/12/2018

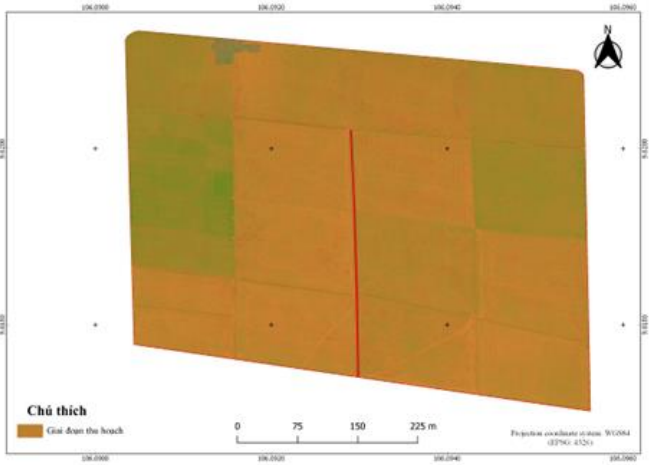

d) Bản đồ lúa ngày 25/01/2019

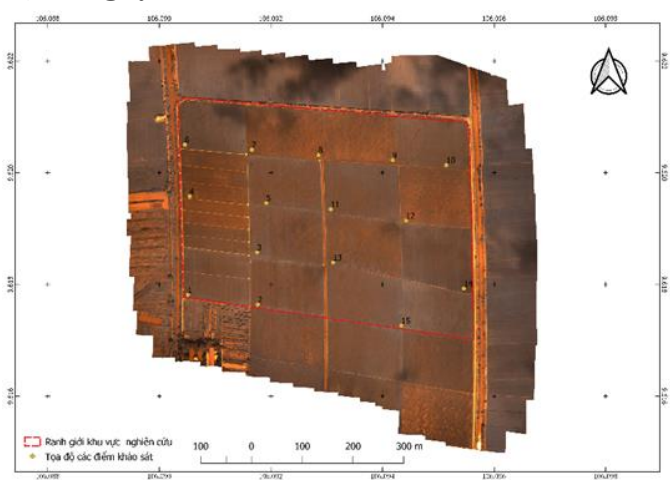

e) Bản đồ vị trí các điểm khảo sát thực địa

Hình 14. Bản đồ lúa nhiễm bệnh được phân tích bằng chỉ số NDVI và bản đồ vị trí các điểm khảo sát thụ̣c địa

Kết quả phân tích diễn biến dịch hại trên lúa thông qua chỉ số NDRE được trình bày thông qua các bản đồ Hình 15 và các biểu đồ Hình 16, cho thấy diện tích lúa bị nhiễm dịch hại gần tương tự như chỉ số NDVI, với tổng diện tích nhiễm bệnh là 11,38 ha, chiếm 48\% khi lúa ở giai đoạn 30 ngày tuổi. Tuy nhiên, ở các giai đoạn lúa 43 ngày và 50 ngày tuổi, diện tích lúa nhiễm bệnh được phân tích bằng chỉ số NDVI và NDRE có sự khác biệt lớn, cụ thể tổng diện tích nhiễm được phân tích bằng chỉ số NDRE tương ứng 5,9 ha và 5,77 ha (chênh lệch so với giá 
trị NDVI tương ứng là 5,43 ha và 5,16 ha). Trong giai đoạn này, diện tích lúa nhiễm dịch hại chủ yếu ở mức độ nhẹ chiếm hơn $68 \%$ diện tích nhiễm bệnh

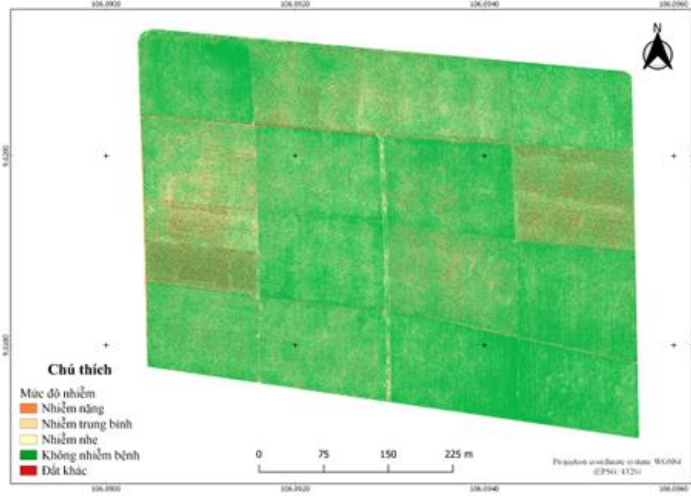

a) Bản đồ lúa bị nhiễm bệnh ngày 22/11/2018

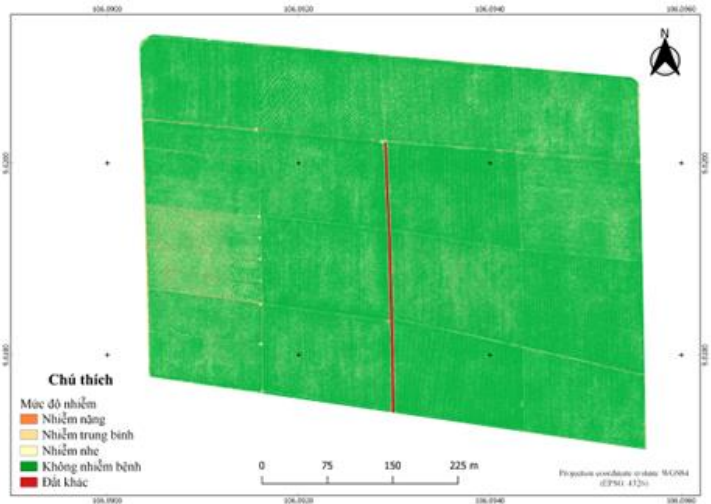

c)Bản đồ lúa bị nhiễm bệnh ngày 12/12/2018 khi lúa 43 ngày và $77 \%$ diện tích nhiễm bệnh khi lúa 50 ngày.

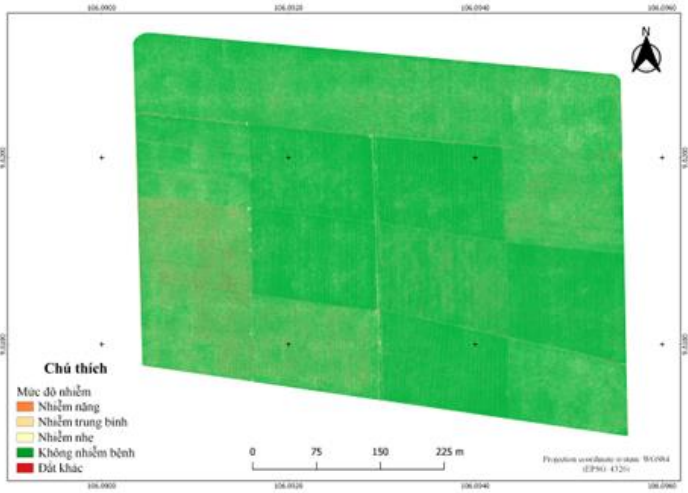

b) Bản đồ lúa bị nhiễm bệnh ngày 5/12/2018

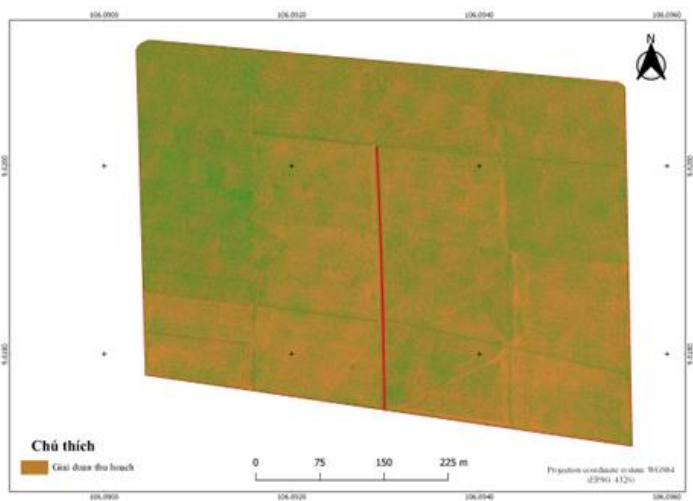

d) Bản đồ lúa ngày 25/01/2019

Hình 15. Bản đồ lúa nhiễm bệnh được phân tích bằng chỉ số NDRE

Thông qua các biểu đồ so sánh diện tích nhiễm dịch hại được phân tích bằng chỉ số NDVI, NDRE và kết quả khảo sát thực địa, được thể hiện trong Hình 16 dễ dàng nhận thấy diện tích nhiễm dịch hại được phân tích bằng chỉ số NDRE và dữ liệu khảo sát thực địa có sự tương đồng cao hơn so với phân tích bằng giá trị NDVI, với hệ số tương quan diện tích tương ứng là $\mathrm{r}=0,97$ và $\mathrm{r}=0,69$.

Ở giai đoạn 30 ngày tuổi, lúa bị nhiễm bệnh ở mức độ trung bình và nhe thì diện tích nhiễm bệnh được phân tích bằng chỉ số NDVI, NDRE và khảo sát thực địa không có sự chênh lệch nhiều, được thể hiện trong Hình 16-a. Tuy nhiên, ở các giai đoạn 43 ngày và 50 ngày, lúa bị nhiễm bệnh chủ yếu ở mức độ nhẹ, thì diện tích nhiễm bệnh được phân tích bằng chỉ số NDVI, NDRE và khảo sát thực địa có sự khác nhau lớn, diện tích nhiễm bệnh được phân tích bằng chỉ số NDRE và khảo sát thực địa lớn hơn so với diện tích nhiễm bệnh được phân tích bằng chỉ số NDVI, được thể hiện trong Hình 16-b, Hình 16c. Vì thế cho thấy, chỉ số NDRE có độ nhạy với lúa nhiễm bệnh cao hơn chỉ số NDVI, đặc biệt khi lúa ở mức độ nhiễm nhẹ, ở giai đoạn đầu phát triển của dịch hại nên chỉ số NDRE có khả năng ứng dụng theo dõi và cảnh báo sớm dịch hại cao hơn. 


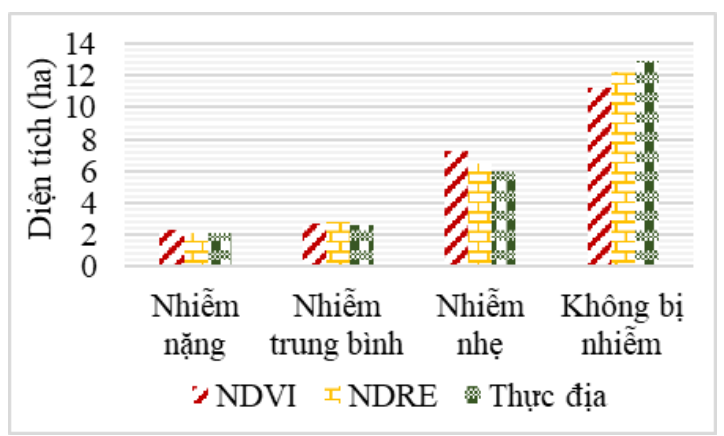

a) Diện tích nhiễm bệnh ngày 22/11/2018

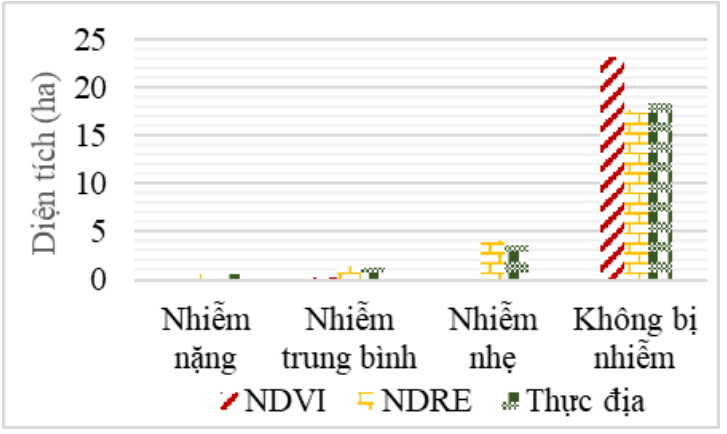

b)Diện tích nhiễm bệnh ngày 05/12/2018

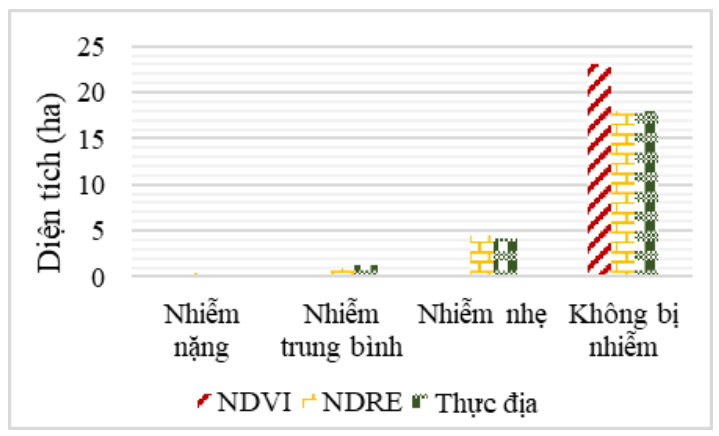

c) Diện tích nhiễm bệnh ngày 12/12/2018

\section{Hình 16. Các biểu đồ so sánh diện tích lúa nhiễm bệnh được phân tích dựa trên chỉ số NDVI, NDRE và dữ liệu khảo sát thực địa}

\section{KẾT LUẬN}

Việc ứng dụng công nghệ UAV trong theo dõi diễn biến dịch hại để đưa ra các cảnh báo sớm đang mang lại nhiều hiệu quả, giúp nhà sản xuất theo dõi tình hình dịch hại kịp thời và chính xác. Nghiên cứu cho thấy chỉ số NDRE có độ nhạy để xác định mức độ nhiễm dịch hại cao hơn chỉ số NDVI, đặc biệt khi dịch hại đang ở mức độ nhiễm trung bình và nhẹ. Vì thế, việc ứng dụng phương pháp phân tích lúa nhiễm dịch hại bằng công nghệ UAV đã giúp phát hiện sớm và chính xác mức độ nhiễm dịch hại, góp phần ngăn chặn và phòng ngừa dịch hại kịp thời, giảm chi phí và nâng cao hiệu quả sản xuất lúa.

Kết quả nghiên cứu cho thấy lúa bị nhiễm dịch hại nhiều nhất ở giai đoạn đẻ nhánh ( 30 ngày tuổi) với tổng diện tích nhiễm là 11,37 ha, trong đó phần lớn diện tích nhiễm ở mức độ trung bình và nặng, trong giai đoạn này lúa chủ yếu bị ảnh hưởng bởi sâu lá và bệnh đạo ôn lá. Trong giai đoạn lúa 43 ngày và 50 ngày tuổi, diện tích nhiễm bệnh đã có xu hướng giảm, chủ yếu ở mức độ nhiễm trung bình và nhẹ.

Tuy nhiên, nghiên cứu này vẫn còn nhiều điểm cần phải cải thiện như số lượng ảnh phải được thu thập cho toàn bộ thời gian canh tác lúa, các số liệu được thu thập ở ngoài đồng phải được chuẩn hóa để có thể đánh giá độ chính xác kết quả phân tích giá trị NDVI và NDRE trên ảnh.

\section{LỜI CẢM TẠ}

Đề tài này được tài trợ bởi Dự án Nâng cấp Trường Đại học Cần Thơ VN14-P6 bằng nguồn vốn vay ODA từ Chính phủ Nhật Bản.

\section{TÀI LIỆU THAM KHẢO}

Aylor, D. E., Boehm, M. T., \& Shields, E. J. (2006). Quantifying aerial concentrations of maize pollen in the atmospheric surface layer using remotepiloted airplanes and Lagrangian stochastic modeling. Journal of Applied Meteorology and Climatology, 45(7), 1003-1015.

Chosa, T., Miyagawa, K., Tamura, S., Yamazak, K., Iiyoshi, S., Furuhata, M., \& Motobayash, K. (2010). Monitoring rice growth over a production region using an unmanned aerial vehicle: Preliminary trial for establishing a regional rice strain. IFAC Proceedings Volumes (IFAC-PapersOnline), 43(26), 178-183.

Duan, B., Fang, S., Zhu, R., Wu, X., Wang, S., Gong, Y., \& Peng, Y. (2019). Remote Estimation of Rice Yield With Unmanned Aerial Vehicle 
(UAV) Data and Spectral Mixture Analysis. Frontiers in Plant Science, 10(26), 204-217.

Barnes E. M., Clarke T. R., Richards S. E., Colaizzi P. D, Haberland J., Kostrzewski M., Waller P., Choi C., Riley E., Thompson T., Lascano R. J., Li H., Moran M. S. (2000). Coincident Detection Of Crop Water Stress, Nitrogen Status And Canopy Density Using Ground-Based Multispectral Data. Proceedings of the Fifth International Conference on Precision Agriculture, 16(19), 1-15.

Filho, F. H. I., Heldens, W. B., Kong, Z., \& De Lange, E. S. (2019). Drones: Innovative technology for use in precision pest management. Journal of Economic Entomology, 113(1), 1-25.

Hunt Jr, E. R., Daughtry, C., Walthall, C., III, J. E., \& Dulaney, W. (2003). Agricultural Remote Sensing using Radio-Controlled Model Aircraft. IEEE Journal of Selected Topics in Applied Earth Observations and Remote Sensing, 7(11), 4566 - 4571.

Sinha J. P., Kushwaha H. L., Kushwaha D., Singh N., \& Purushottam M. (2016). Prospect of Unmanned Aerial Vehicle (UAV) Technology for Agricultural Production. Agricultural and Food Engineering Department, 53-66.

Kameyama, S., \& Sugiura, K. (2020). Estimating Tree Height and Volume Using Unmanned Aerial Vehicle Photography and SfM Technology, with Verification of Result Accuracy. Drones, 4(2), 19.

Kitpo, N., \& Inoue, M. (2018). Early Rice Disease Detection and Position Mapping System using Drone and IoT Architecture. PublisherInstitute of Electrical and Electronics Engineers Inc, 1-5.

Matese, A., Toscano, P., Di Gennaro, S. F., Genesio, L., Vaccari, F. P., Primicerio, J., Belli, C., Zaldei, A., Bianconi, R., \& Gioli, B. (2015). Intercomparison of UAV, aircraft and satellite remote sensing platforms for precision viticulture. Remote Sensing, 7(3), 2971-2990.
MicaSense (2018). Hướng dẫn của nhà cung cấp sản phẩm. Truy cập ngày 26/2/2020 từ https://micasense.com/

Ngadiman, N., Kaamin, M., Sahat, S., Mokhtar, M., Ahmad, N. F. A., Kadir, A. A., \& Razali, S. N. M. (2018). Production of orthophoto map using UAV photogrammetry: A case study in UTHM Pagoh campus. AIP Conference Proceedings, 1-6.

Norasma, C. Y. N., Abu Sari, M. Y., Fadzilah, M. A., Ismail, M. R., Omar, M. H., Zulkarami, B., Hassim, Y. M. M., \& Tarmidi, Z. (2018). Rice crop monitoring using multirotor UAV and RGB digital camera at early stage of growth. IOP Conference Series: Earth and Environmental Science, 158-169.

Norasma, C. Y. N., Fadzilah, M. A., Roslin, N. A., Zanariah, Z. W. N., Tarmidi, Z., \& Candra, F. S. (2019). Unmanned Aerial Vehicle Applications in Agriculture. IOP Conference Series: Materials Science and Engineering, 692-702.

Yacine B., Pierre B., Thuy N. X., Claire G., Carl B., Louis L., \& Philippe V. (2018). Pest Detection on UAV Imagery using a Deep Convolutional Neural Network. Remote Sensing, 52(19), 17-31.

Sow, M. T., (2014). Using ANOVA to Examine the Relationship between Safety \& Security and Human Development. Journal of International Business and Economics, 2(4), 101-106.

Strange, R. N., \& Scott, P. R. (2005). Plant Disease: A Threat to Global Food Security. Annual Review of Phytopathology, 43(1), 83-116.

Swain, K., Jayasuriya, H., \& Salokhe, V. (2007). Suitability of low-altitude remote sensing images for estimating nitrogen treatment variations in rice cropping for precision agriculture adoption. Journal of Applied Remote Sensing, 1(1), 254-276.

Tucker, C. J., (1979). Red and photographic infrared linear combinations for monitoring vegetation. Remote Sensing of Environment, 8(3), 127-150. 